\title{
Combined effects of trapped energetic ions and resistive layer damping on the stability of the resistive wall mode
}

\author{
Yuling He, ${ }^{1}$ Yueqiang Liu, ${ }^{2,3,4, a)}$ Yue Liu, ${ }^{1, a)}$ Chao Liu, ${ }^{1}$ Guoliang Xia, ${ }^{1}$ Aike Wang, ${ }^{3}$ \\ Guangzhou Hao, ${ }^{3} \mathrm{Li} \mathrm{Li}^{5}$ and Shaoyan $\mathrm{Cui}^{6}$ \\ ${ }^{1}$ Key Laboratory of Materials Modification by Laser, Ion and Electron Beams, Ministry of Education, \\ School of Physics and Optoelectronic Technology, Dalian University of Technology, Dalian 116024, China \\ ${ }^{2}$ CCFE, Culham Science Centre, Abingdon OX14 3DB, United Kingdom \\ ${ }^{3}$ Southwestern Institute of Physics, P.O. Box 432, Chengdu 610041, China \\ ${ }^{4}$ Department of Earth and Space Science, Chalmers University of Technology, SE-412 96 Gothenburg, Sweden \\ ${ }^{5}$ College of Science, Donghua University, Shanghai 201620, China \\ ${ }^{6}$ School of Mathematics and Statistics Science, Ludong University, Yantai 264025, China
}

(Received 16 July 2015; accepted 17 December 2015; published online 13 January 2016)

\begin{abstract}
A dispersion relation is derived for the stability of the resistive wall mode (RWM), which includes both the resistive layer damping physics and the toroidal precession drift resonance damping from energetic ions in tokamak plasmas. The dispersion relation is numerically solved for a model plasma, for the purpose of systematic investigation of the RWM stability in multi-dimensional plasma parameter space including the plasma resistivity, the radial location of the resistive wall, as well as the toroidal flow velocity. It is found that the toroidal favorable average curvature in the resistive layer contributes a significant stabilization of the RWM. This stabilization is further enhanced by adding the drift kinetic contribution from energetic ions. Furthermore, two traditionally assumed inner layer models are considered and compared in the dispersion relation, resulting in different predictions for the stability of the RWM. (C) 2016 AIP Publishing LLC.

[http://dx.doi.org/10.1063/1.4939806]
\end{abstract}

\section{INTRODUCTION}

It is well known that the external kink mode and the tearing mode are dangerous macroscopic magnetohydrodynamic (MHD) instabilities in a tokamak device, such as the International Thermonuclear Experimental Reactor (ITER). ${ }^{1}$ In order for a tokamak device to achieve high $\beta$ (ratio of the plasma to the magnetic pressures) plasmas and at the same time maintaining a long time or steady state discharge, stabilization of these MHD instabilities is one of the crucial issues. In this work, we shall focus on investigating the stability of the external kink mode, which often causes the socalled "hard" beta limit for the tokamak plasma discharges, compared to the "soft" beta limit imposed by the onset of the (neoclassical) tearing mode.

The external kink modes can fortunately be completely stabilized by a perfectly conducing wall which surrounds the plasma and is located sufficiently close to the plasma surface, up to a certain beta limit. ${ }^{2}$ In reality, the wall almost always has a finite conductivity, allowing the leakage of the radial magnetic flux perturbation at a longer time scale, and thus an instability will eventually grow. In this situation, the fast growing external kink mode becomes a slowly growing instability called the resistive wall mode (RWM). ${ }^{2}$ The growth time of the RWM is in the same order as the eddy current diffusion time, while the magnetic field perturbation penetrates through the resistive wall. For the concept of advanced tokamaks, which aims at a steady state operation,

\footnotetext{
${ }^{\text {a) }}$ Authors to whom correspondence should be addressed. Electronic addresses:

Yueqiang.Liu@ccfe.ac.uk and liuyue@dlut.edu.cn
}

the RWM is the most dangerous instability, which needs to be either stable or stabilized.

The mode can be described by different plasma models. In many numerical and analytical studies, ${ }^{3-10}$ the plasma is generally assumed to be ideal, i.e., with vanishing plasma resistivity. Early work has shown that the plasma toroidal flow, with certain energy dissipation channel(s) being present inside the plasma, can help to open up a stability window by varying the radial location of the resistive wall. ${ }^{1-13}$ For example, the shear Alfven or sound wave resonance damping model yields a critical plasma rotation frequency of typically a few percent of the Alfven frequency. ${ }^{7,11,13}$ Recent work mainly focuses on the drift kinetic damping on the RWM. ${ }^{14-21}$ The drift kinetic theory seems to produce a better agreement with experiments on the RWM stability, in terms of both the threshold value of the plasma rotation frequency for the RWM stabilization and the resonant field amplification (RFA) observed in high beta plasmas. ${ }^{22-25}$

One key element in the drift kinetic model is the resonance between the mode and the toroidal precession drift motion of trapped thermal particles. It was found that this resonant damping can fully stabilize the RWM in a slowly rotating plasma. ${ }^{14-16,18}$ The presence of the precessional drift resonance of energetic ions, again with the assumption of ideal plasma, on the other hand, can either enhance the stability of the RWM ${ }^{26}$ or trigger a new branch of instability termed as the fishbonelike bursting mode (FLM). Such an instability, with the eigenmode structure of an external kink mode, can occur when the energetic particles' (EPs') beta exceeded a critical value.

The role of the plasma resistivity on the RWM stability has been less well exploited. In the early work, ${ }^{27,28}$ Finn 
showed that a slow plasma rotation, combined with a resistive layer near the mode's rational surface, can lead to the wall mode stabilization. Subsequently, the beta limit for the $n=1$ mode is set by the resistive-wall-tearing mode (RWTM). ${ }^{13}$ Reference 29 showed that the toroidal curvature effect associated the resistive layer may stabilize the RWM, but the stability window is narrow. All the aforementioned work assumes a fluid model for the mode. In this work, we perform a systematic investigation of the RWM stability, by considering both the resistive layer physics and the drift kinetic damping from energetic ions. This expands the investigation reported in a recent work, ${ }^{30}$ in terms of both the dispersion relation for the mode and the numerical results.

Section II presents the details of our models, for both the RWM and the plasma equilibrium that we use in this work. Section III reports key results obtained from numerical solution of the proposed dispersion relations for the RWM. Section IV draws conclusion.

\section{MODELS}

\section{A. Plasma equilibrium model}

We choose an equilibrium model as shown in Fig. 1, for a large aspect ratio plasma with circular poloidal cross section. The plasma is in the region $0<r<a$, in terms of the minor radius $r$. A conducting wall is located at $r_{w}$ in the vacuum region. We introduce the inverse aspect ratio $\varepsilon=r / R$, with $R$ being the major radius. The plasma equilibrium pressure is a constant $P_{0}$. The plasma toroidal current density and the safety factor are also largely constants, $J_{0}$ and $q_{0}$, respectively, except within a narrow resistive layer near the plasma boundary. Within this narrow layer, we assume that the equilibrium current vanishes, and thus, the q-profile increases, allowing the existence of a rational surface $(q=2$ in this work) inside the plasma. Here, we considered only one rational surface. Thus, the coupling effect between different rational surfaces is neglected. Such a coupling may not be strong if two rational surfaces are located far from

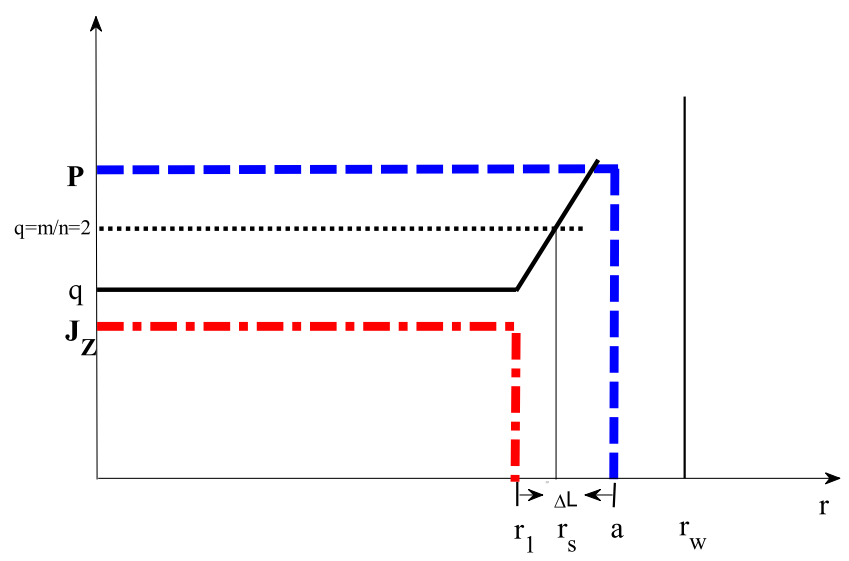

FIG. 1. The equilibrium profiles of the plasma pressure $P(r)$, the current density $J_{z}(r)$, and the safety factor $q(r)$ as assumed in the cylindrical model. One rational surface $\left(r=r_{s}\right)$, with $\mathrm{q}=\mathrm{m} / \mathrm{n}=2 / 1$, is located just inside the plasma boundary $(r=a)$. The width of the resistive layer is assumed to be much smaller than the plasma minor radius, $\Delta L \ll a$. A resistive wall is located at $r=r_{w}=b$. each other. However, in certain conditions, particularly near the plasma edge region, the interaction between closely located multiple rational surfaces may be indeed significant. Such a coupling effect, combined with the drift kinetic effect from the trapped EPs, will be numerically investigated using the MARS-K code $^{15}$ in the future. We consider a plasma with finite resistivity in a layer, of width $\Delta L$ which is much smaller than the plasma minor radius $a$, near the rational surface $\left(r=r_{s}\right)$. Outside the resistive layer, the plasma is still described by the ideal MHD equations.

\section{B. Dispersion relation for the RWM}

There are typically two ways to construct a dispersion relation for the RWM. One way is to rely on the matching condition for the tearing index $\Delta^{\prime}$ between the external ideal region and the internal resistive-inertial region. ${ }^{29,31}$ The other is based on the extended energy principle. ${ }^{12,14,32,33}$ Within the fluid approximation, it can be shown that these two approaches are equivalent. However, with the inclusion of the drift kinetic effects, it is more convenient to follow the energy principle approach, since the drift kinetic energy perturbation can often be readily evaluated (either analytically or computationally). This approach, on the other hand, requires evaluation of the fluid energy dissipation in the resistive-inertial layer.

In the following, we show one simple example of derivation of the layer energy dissipation, mainly to demonstrate the linear relation between the perturbed energy and the inner tearing index. We consider the equilibrium with vanishing pressure gradient; thus, the so-called constant- $\psi$ approximation is valid.

We define $x=r-r_{s}$ as our independent radial variable, and the safety factor is $\mathrm{q}\left(r_{s}\right)=m / n$ at the location of the rational surface, where $m$ and $n$ are the poloidal and the toroidal mode numbers, respectively $(m=2$ and $n=1$ throughout this work). All perturbed quantities are taken to vary as $f_{1}=f(\mathbf{x}) \exp [i(m \theta-n \phi)+\gamma t]$. The linearized equations of the momentum equation and Faraday's law in the resistive layer can be expressed as ${ }^{34}$

$$
\begin{gathered}
\psi_{1}(x)+\hat{s} n x \xi(x) / m=r_{s}^{2} \psi_{1}^{\prime \prime}(x) /\left(\gamma \tau_{R}\right), \\
\left(\gamma \tau_{A}\right)^{2} \xi^{\prime \prime}(x)=\hat{s} x \psi_{1}^{\prime \prime}(x) n m / r_{s}^{2},
\end{gathered}
$$

where the perturbed poloidal flux function $\psi_{1}$ is simply related to the plasma displacement $\vec{\xi}$ via Eq. (2). $\gamma=\hat{\gamma}-i \omega_{r}$ is the eigenvalue of the mode, $\hat{s}=r_{s} q^{\prime} / q$ is the magnetic shear at the rational surface, $\tau_{A}=R \sqrt{\rho} / B_{0}$ is the characteristic Alfven time, and $\tau_{R}=\mathrm{r}_{s}^{2} \mu_{0} / \eta$ is the resistive diffusion time. $S=\tau_{R} / \tau_{A}$ is the magnetic Lundquist number. For $m>1$ mode, it is known that the energy perturbation associated with the layer is proportional to $\Delta^{\prime},{ }^{34}$ which is defined as the logarithmic jump of the radial derivative of $\psi_{1}$ across the rational surface, for the case described above. In a more general case, it can be rigorously shown that ${ }^{30}$

$$
\delta W_{R L}^{m>1}=\frac{4 \pi}{m} \frac{\left(m-n q_{0}\right)^{2}}{q_{0}^{2}} \frac{r_{s}}{2 m} \Delta^{\prime}\left(\gamma+i \omega_{0}\right),
$$


where a uniform radial profile for the safety factor $q, q=q_{0}$, has been assumed, and $\omega_{0}$ is the toroidal rotation frequency of the plasma which Doppler shifts the mode frequency.

Unlike Ref. 30, here we shall consider two layer models for $\Delta^{\prime}$, which have been conventionally assumed in literatures for the RWM study. ${ }^{13,27-29,31}$ The first is the tearing mode dispersion relation including the toroidal favorable curvature effect. ${ }^{35}$ For the large aspect ratio circular plasma, as considered in this work, the $\Delta^{\prime}$ is conveniently written as ${ }^{36}$

$$
\Delta_{1}^{\prime}=2.12 A\left(\frac{\gamma+i \omega_{0}}{\omega_{A}}\right)^{5 / 4}\left[1-\frac{\pi}{4} D_{R} B\left(\frac{\gamma+i \omega_{0}}{\omega_{A}}\right)^{-3 / 2}\right]
$$

where the coefficients $A \equiv 2 \pi \Gamma(3 / 4) / \Gamma(1 / 4)(n \hat{s})^{-1 / 2}$ $\left(1+2 q_{r_{s}}{ }^{2}\right)^{1 / 4} S^{3 / 4}$ and $B \equiv(n \hat{s})\left(1+2 q_{r_{s}}{ }^{2}\right)^{-1 / 2} S^{-1 / 2}$ are all evaluated at the mode rational surface. $\omega_{A}$ and $D_{R}$ are the Aflven frequency and the resistive interchange index, respectively. The last term from Eq. (4a), $\frac{\pi}{4} D_{R} B\left(\frac{\gamma+i \omega_{0}}{\omega_{A}}\right)^{-3 / 2}$, originates from the favorable average magnetic curvature in the layer region, ${ }^{35}$ often referred to as the GGJ effect. In the limit of a vanishing $D_{R}$ value, Eq. (4a) recovers the tearing mode dispersion relation at the constant- $\psi$ approximation (apart from the inertial enhancement factor $1+2 q_{r_{s}}^{2}$ ). The addition of the GGJ correction in the inner $\Delta^{\prime}$ model is ad$h o c$ with respect to our equilibrium model. However, this approach is not completely unreasonable, since the inner layer $\Delta^{\prime}$, in theory, is determined by the local equilibrium pressure gradient exactly at the rational surface, thus allowing flexibility in the choice of the radial profile for the equilibrium pressure outside the rational surface. This is largely why the same ad-hoc approach was taken in literatures such as Ref. 29. In further numerical investigations in this work, we shall vary the value of $D_{R}$, in order to switch on/off the GGJ effect, as well as to change the strength of the GGJ stabilization. This is despite the fact that our equilibrium model, which is designed to allow accurate derivation of the plasma fluid potential energy perturbations, corresponds to the case of $D_{R}=0$.

The second model for $\Delta^{\prime}$ follows the resistive kink dispersion relation $^{37}$

$\Delta_{2}^{\prime}=-\frac{\pi}{\mathbf{8}}\left[\frac{\gamma+i \omega_{0}}{\omega_{A}}\right]^{5 / 4} S^{3 / 4} \frac{\left.\Gamma\left[\mathbf{1} / \mathbf{4}\left(\frac{\gamma+i \omega_{0}}{\omega_{A}}\right)^{3 / 2} S^{1 / 2}-\mathbf{1}\right)\right]}{\left.\Gamma\left[\mathbf{1} / \mathbf{4}\left(\frac{\gamma+i \omega_{0}}{\omega_{A}}\right)^{3 / 2} S^{1 / 2}+\mathbf{5}\right)\right]}$.

The resistive kink dispersion relation has been used in the RWM study in Refs. 13 and 31. We point out that this $\Delta^{\prime}$ does not include the favorable toroidal curvature stabilization effect. This will have consequences on the eventual RWM stability prediction, compared to that by the tearing layer model (4a).

The energy dissipation associated with the resistive layer, Eqs. (3) and (4), allows us to construct a dispersion relation for the RWM including all relevant effects, based on the energy balance principle

$$
D=-i\left(\Omega+\Omega_{0}\right) \omega_{d s} \tau_{w}^{*}+\frac{\delta \mathrm{W}^{\infty}+\delta W_{K 0}+\delta W_{R L}}{\delta \mathrm{W}^{b}+\delta W_{K 0}+\delta W_{R L}}=0 .
$$

Here, a re-normalization has been adopted for the mode's eigenvalue, $\frac{\gamma+i \omega_{0}}{\omega_{A}} \equiv-i \frac{\omega_{d s}}{\omega_{A}} \Omega$, mainly for the convenience in evaluating the drift kinetic energy perturbation $\delta W_{K 0}$ due to trapped fast ions. The new normalization factor, $\omega_{d s}$, is the toroidal precession frequency of these fast ions at the birth energy, $\Omega_{0}=\omega_{0} / \omega_{d s} . \quad \tau_{w}^{*}=\mu_{0} \sigma b d\left[1-(b / a)^{-2 m}\right] /(2 m)$ is the wall eddy current decay time, with $\mathrm{d}, \sigma$, and $\mu_{0}$ being the wall thickness, the wall conductivity, and the permeability of free space, respectively. $\delta \mathrm{W}^{\infty}$ and $\delta \mathrm{W}^{b}$ are the perturbed fluid potential energies (includes both the plasma and vacuum contributions) with an ideal wall at infinity and at $r=b$, respectively.

The dispersion relation (5) follows the approach of Ref. 12, where the (generic) dissipation term is now replaced by the combined energy term associated with the drift kinetic energy and the resistive layer damping energy. In the presence of the drift kinetic effects but without the resistive layer damping physics, the energy principle has been derived in literatures, e.g., Ref. 38. Vice versa, in the presence of the resistive layer damping but without the drift kinetic effects, a matching procedure between the outer and the inner layer solutions is often applied to construct the resistive plasma RWM dispersion relation, ${ }^{29}$ which is shown to be equivalent to the energy perturbation based form. ${ }^{30}$ However, no rigorous derivation is available for Eq. (5), when both the kinetic effects and the resistive layer damping physics are present in the model, though similar approach has also been used in studying the resistive fishbone mode. ${ }^{39}$

For the equilibrium model defined in this work, the fluid and drift kinetic energy can be analytically evaluated ${ }^{20,40}$

$$
\begin{gathered}
\delta W^{\infty}=\frac{-4 \pi}{m} \frac{(m-n q)^{2}}{q^{2}}\left(\frac{1}{m-n q}-1\right), \\
\delta W^{b}=\frac{-4 \pi}{m} \frac{(m-n q)^{2}}{q^{2}}\left(\frac{1}{m-n q}-\frac{1}{1-b^{-2 m}}\right), \\
\delta W_{K 0}=12 \pi\left(1-\frac{\alpha_{0} B_{0}}{2}\right)^{2} \frac{\beta_{h} R}{K a}\left\{(\hat{A}-\hat{B}) \frac{2}{7} \Omega \ln \left(1-\frac{1}{\Omega}\right)\right. \\
-\frac{2}{7}\left(\hat{A}+\frac{2}{5} \hat{B}\right) \Omega\left[2\left(\frac{1}{5 \Omega}+\frac{1}{3 \Omega^{2}}+\frac{1}{\Omega^{3}}\right)\right. \\
\left.\left.-\frac{1}{\Omega^{3}} \frac{1}{\sqrt{\Omega}} \ln \left(\frac{1+\sqrt{\Omega}}{1-\sqrt{\Omega}}\right)\right]\right\}+M,
\end{gathered}
$$

where $\omega_{d s}=(2 \mathrm{E}-\mathrm{K}) E_{m} q_{0} /\left[\mathrm{K} a \omega_{c} R\right]$ is, as mentioned before, the precession drift frequency of the trapped EPs at birth energy. $\mathrm{E}$ and $\mathrm{K}$ are the complete elliptic integrals of the first and second kinds, respectively, $m_{h} E_{m}\left(\equiv E_{b}\right)$ and $\omega_{c}$ are the birth energy and the cyclotron frequency of the trapped EPs, respectively. $\beta_{h}=c_{\beta} \int r d r d \alpha d E K_{b} E f\left(\alpha=\mu m_{h} / E_{k}, \mu=v_{\perp}^{2} / 2 B\right.$, $E_{k}=m_{h} E$, and $K_{b}=\int_{-\theta_{b}}^{\theta_{b}}(1-\alpha B)^{-1 / 2} d \theta / \pi$, with $\theta_{b}$ being the 
turning point of the trapped EPs) is the perpendicular beta of the trapped EPs with $c_{\beta}=\pi N_{p} \mu_{0} m_{h} /\left(R B_{0} r_{1}^{2}\right), N_{p}$ being the total number of EPs, with a slowing-down equilibrium distribution in the particle energy space and a mono-pitch angle in velocity distribution, i.e., $f \propto n_{0} E^{-1} \delta\left(\alpha-\alpha_{0}\right)$. Such distribution is normally a reasonable description of EPs induced by neutral beam injections. The coefficients $\hat{A}, \hat{B}$, and $M$ are derived and listed in Ref. 40. All of the perturbed energy terms, including the layer dissipation (3), are normalized by a factor $\pi B^{2} r_{1}^{4} m^{2} / 2 R \mu_{0} F_{0}^{2}$, where $F_{0}=\left(m-n q_{0}\right) r_{1} /\left(R q_{0}\right)$.

Note that the drift kinetic energy $\delta W_{K 0}$ covers the nonadiabatic contribution only. The adiabatic contribution is absorbed into the fluid potential energy terms following the conventional approach, which is based on the understanding that the total plasma equilibrium pressure is contributed by both the thermal particle pressure and the EPs pressure.

The fluid rotation enters into the internal $\Delta^{\prime}$ (for both the GGJ model and the resistive kink model) via the Doppler shift. This procedure is well justified and accepted in literatures (e.g., Ref. 29), by assuming that the inner solution (effectively the magnetic island) is rotating together with the plasma, whilst the outer ideal solution is not affected by the plasma flow. The latter is an approximation but is normally well satisfied as long as the flow is well sub-sonic.

\section{NUMERICAL RESULTS}

We numerically solve the dispersion relation (5) with the following choices of the basic plasma parameters: $a=1 \mathrm{~m}$, $R=3 \mathrm{~m}, r_{1}=0.98 \mathrm{~m}, B_{0}=2.3 \mathrm{~T}, q_{0}=1.42$, and $\beta=0.055$. The pitch angle of the trapped EPs is $\alpha_{0} \mathrm{~B}_{0}=0.98$. The EPs' birth energy is $E_{b}=85 \mathrm{keV}$. The wall (surface) conductivity is assumed to be $\sigma=10^{6} \Omega^{-1} \mathrm{~m}^{-1}$, and the wall thickness is $d=0.01 \mathrm{~m}$. The plasma density is $n_{0}=10^{20} \mathrm{~m}^{-3}$. These parameters are chosen mainly to ensure a typical RWM regime (e.g., $\delta W^{\infty}<0$ and $\delta W^{b}>0$ ). With these parameters, we calculate the precessional drift frequency of fast ions as $\omega_{d s}$

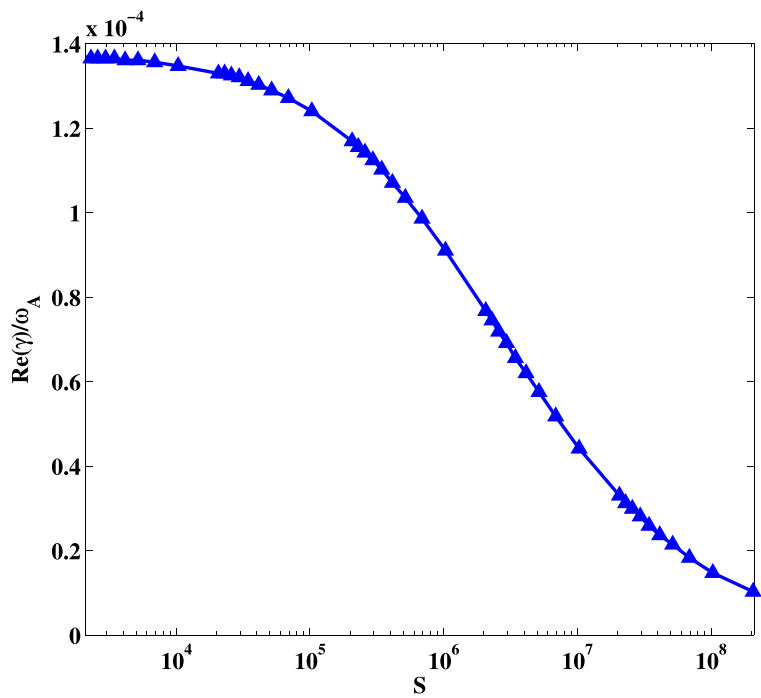

FIG. 2. The growth rate of the $n=1$ fluid RWM versus the Lundquist number $S$. The resistive wall is assumed to be located at $b / a=1.1$. Neither the kinetic damping from trapped EPs nor the favorable average curvature (GGJ) stabilization is included here.
$=7.5 \times 10^{3} \mathrm{rad} / \mathrm{s}=4.41 \times 10^{-3} \omega_{A}$. Note that we assume an aspect ratio of 3 here, for the purpose of representing a typical conventional tokamak case, even though the theory is derived at the large aspect ratio approximation.

This work aims at a systematic investigation of various physics effects entering into the dispersion relation (5). The key physics are the GGJ stabilization due to the resistive layer, the drift kinetic damping due to EPs, and the comparison of predictions of the RWM stability while following two different models (4a) and (4b) for the resistive layer. These effects will be studied in Subsections III A-III D, beginning with a purely fluid case without the GGJ effect.

The parameter space, which we explore while investigating the above physics effects, includes the plasma resistivity (the Lundquist number $S$ ), the radial location of the resistive wall $(b / a)$ as well as the toroidal rotation frequency of the plasma $\left(\Omega_{0}\right)$.
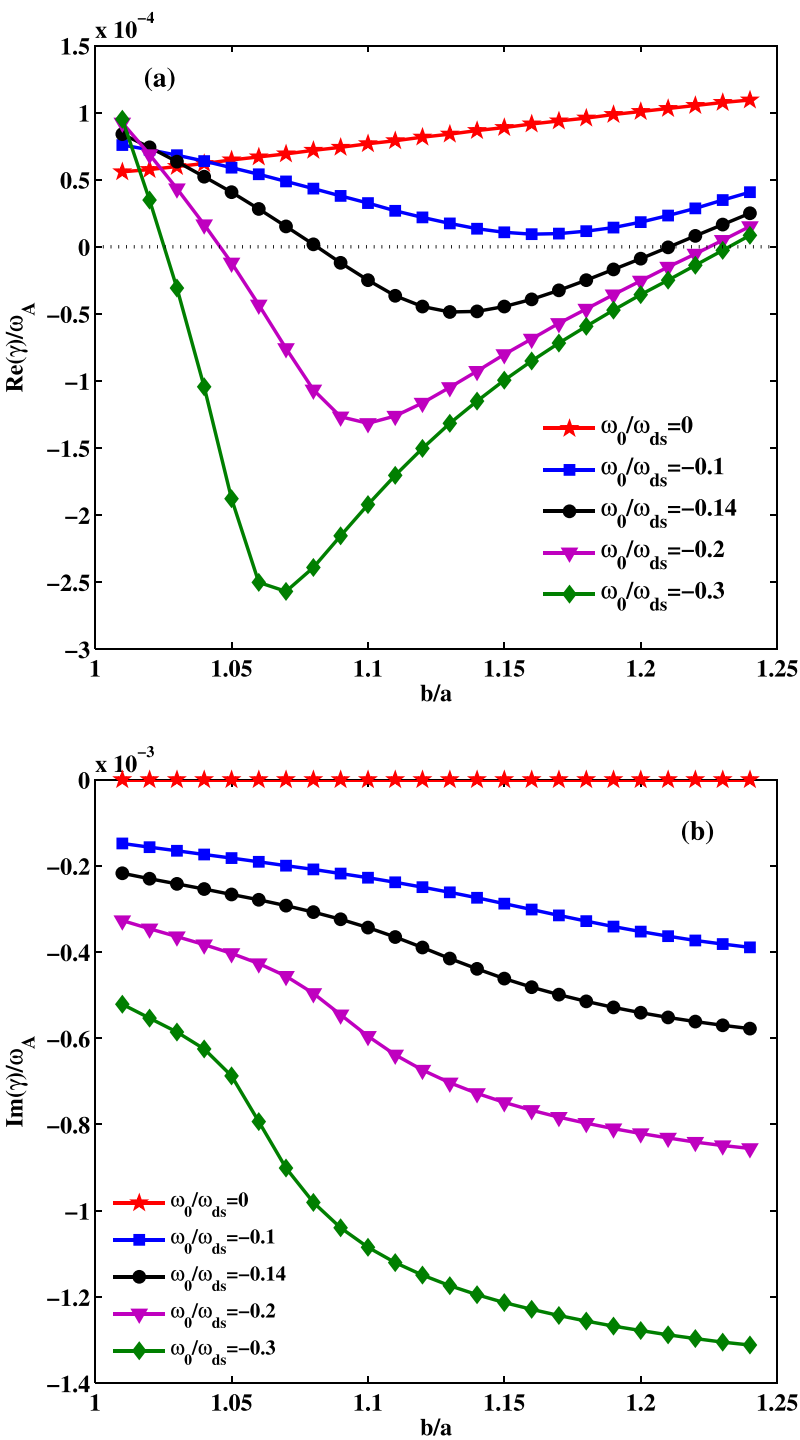

FIG. 3. The (a) growth rate and (b) real frequency of the $n=1$ RWM versus the radial position of the resistive wall, for various choices of the plasma rotation frequency $\left(0<\left|\omega_{0} / \omega_{d s}\right| \leq 0.3\right)$. Here $\omega_{d s}=4.41 \times 10^{-3} \omega_{A}$ is a normalization factor. The Lundquist number is chosen to be $S=2.07 \times 10^{6}$. Neither the kinetic damping from trapped EPs nor the favorable average curvature (GGJ) stabilization is included. 


\section{A. Resistive tearing model without the GGJ effect}

In this subsection, we use the tearing layer model (4a) for $\Delta^{\prime}$ neglecting the GGJ effect $\left(D_{R}=0\right)$. The drift kinetic contribution from EPs is also neglected. This leads to a classical situation where the RWM is destabilized by the plasma resistivity, in the absence of the plasma flow. This is demonstrated in Fig. 2, where we plot the growth rate of the RWM, normalized by the Alfven frequency, versus the Lundquist number $S$. The mode is a purely growing instability in this case.

The presence of a finite toroidal flow generally tends to stabilize the mode, as shown in Fig. 3. Here, the eigenvalue-both (a) real and (b) imaginary parts-are traced with varying wall distance while fixing the Lundquist number at $S=2.07 \times 10^{6}$, for a set of toroidal rotation frequencies $0<\left|\omega_{0} / \omega_{d s}\right|<0.3$. Note that the plasma rotation is chosen in the counter-current direction here, following the same convention as in Ref. 40. The stability (a) of the fluid
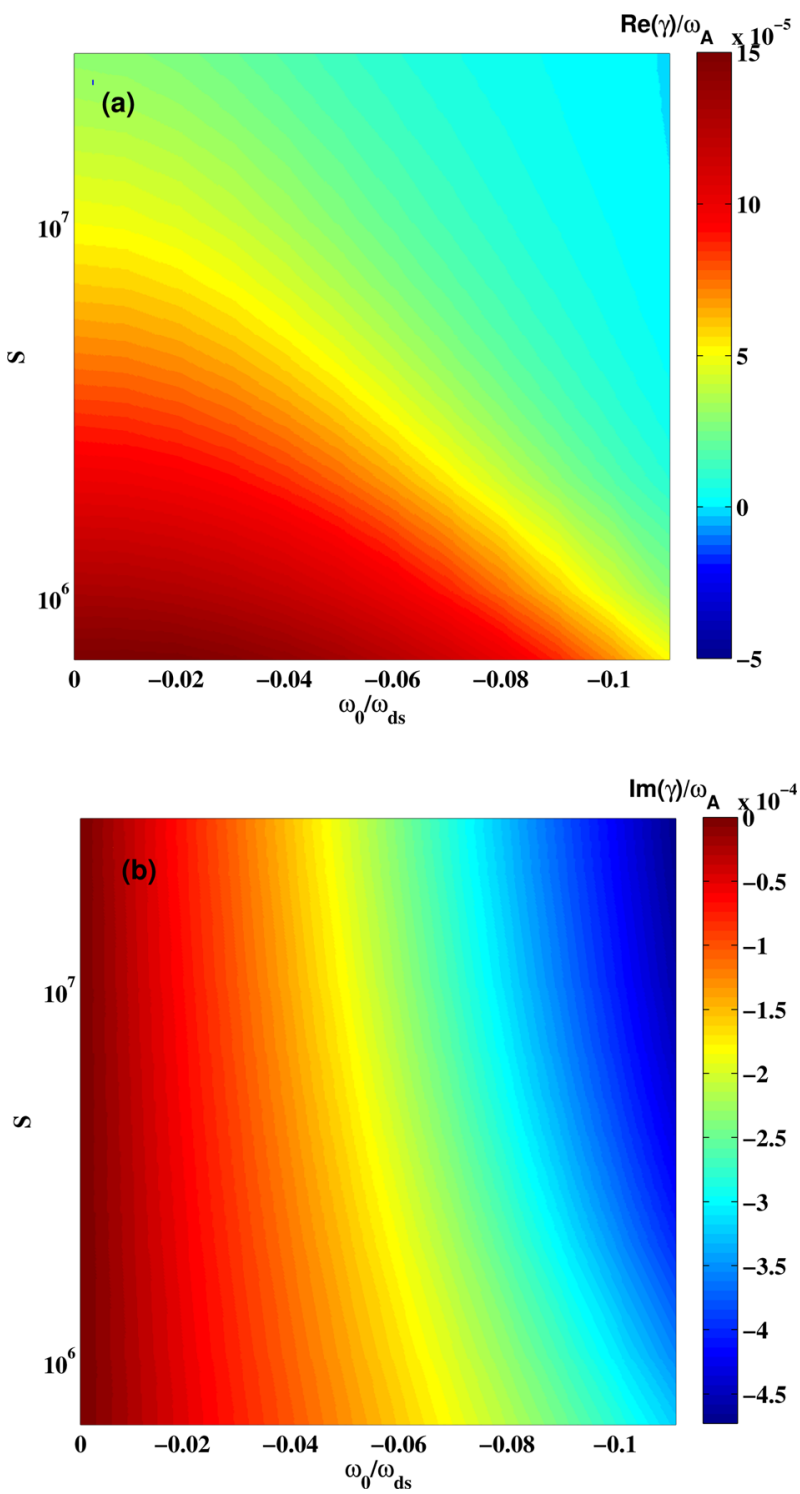

FIG. 4. The (a) growth rate and (b) real frequency of the $n=1$ RWM plotted on 2D parameter space of the plasma rotation frequency and the Lundquist number $S$, with a fixed wall radius at $b / a=1.2$. Neither the kinetic damping from trapped EPs nor the favorable average curvature (GGJ) stabilization is included here.
RWM is not affected by the sign of the rotation frequencyonly the real frequency of the mode (b) switches sign with the change of the flow direction.

A full stabilization of the mode is achieved at sufficiently fast flow, and within a window in $b / a$. This opening of the stability window is similar to that observed, assuming the ideal MHD model. ${ }^{11}$ A minimal flow speed is needed in order to open the stability window, whose size then increases with the flow speed. At a sufficiently fast flow, $\omega_{0} / \omega_{d s}=-0.3$ (equivalent to $\left|\omega_{0}\right| / \omega_{A} \approx 0.13 \%$, the mode is completely stable at a large variation of the wall minor radius. The mode rotates slowly with respect to the resistive wall, when the wall is close to the plasma surface, as shown in Fig. 3(b). However, when the wall is sufficiently far away from the plasma, the mode's frequency tracks well the plasma rotation frequency. In other words, the mode is locked to the plasma in these cases, similar to the prediction of the ideal fluid theory ${ }^{3,12}$

In order to better understand the interplay between the plasma flow and the plasma resistivity on the stability of the
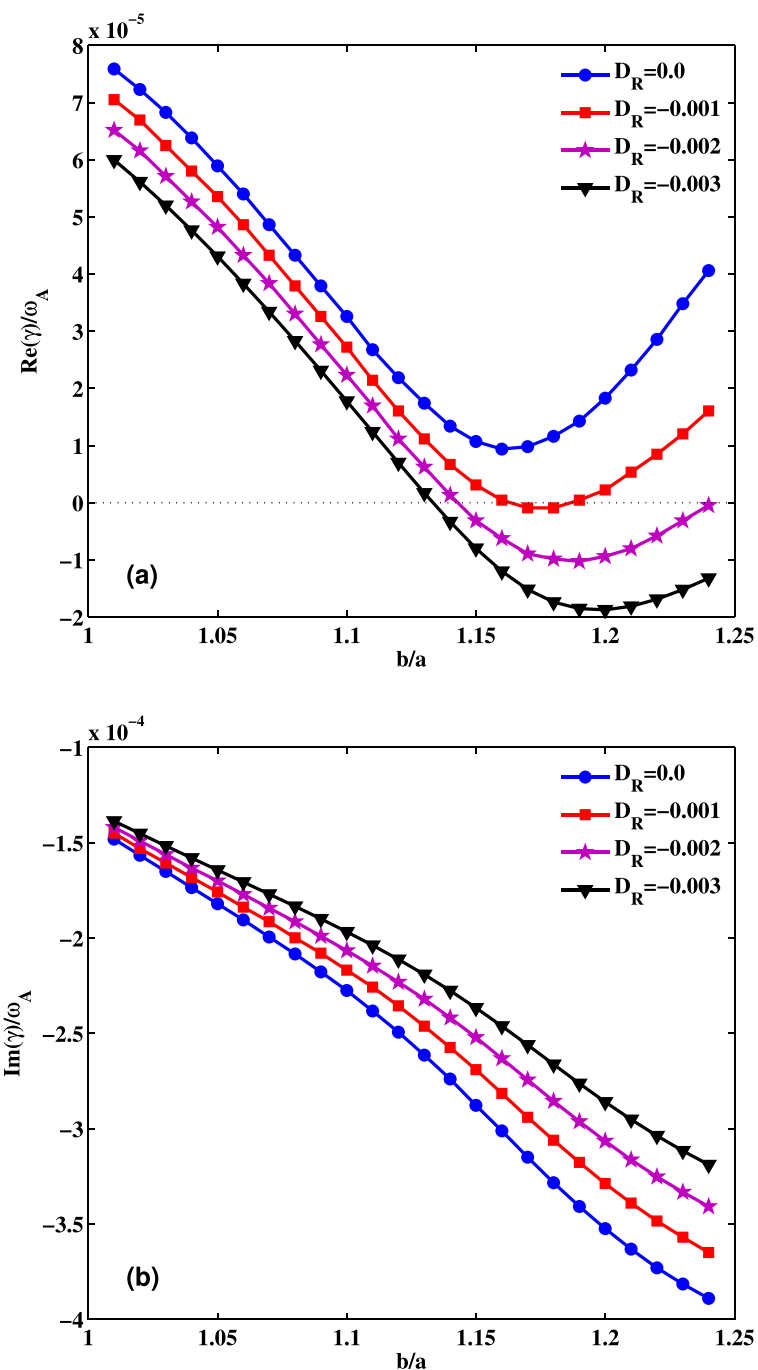

FIG. 5. The (a) growth rate and (b) real frequency of the $n=1$ RWM versus the minor radius $(b / a)$ of the resistive wall. The favorable curvature stabilization effect is included by assuming a finite value for the resistive interchange index $D_{R}$. The plasma rotation frequency is fixed at $\omega_{0} / \omega_{d s}=-\mathbf{0 . 1}$, and the Lundquist number fixed at $S=2.07 \times 10^{6}$. No drift kinetic damping from trapped EPs is included. 
RWM, we carry out a 2D scan of the mode's eigenvalue by varying both $\omega_{0}$ and the Lundquist number $S$, while fixing the wall radius at $b=1.2 a$. The growth rate of the mode, calculated as the solution of the dispersion relation (5) and shown in Fig. 4(a), decreases with increasing the Lundquist number increasing, as well as increasing the flow speed, showing a synergistic effect of small plasma resistivity and fast flow on the stabilization of the RWM, within the fluid assumptions (no GGJ effect). Note that no full stabilization of the mode is achieved with the rotation frequency range shown in the figure. The mode rotating frequency, shown in Fig. 4(b), is generally not a strong function of the plasma resistivity, but is sensitive to the flow velocity.

\section{B. Resistive tearing model with the GGJ effect}

As the next step of investigation, we include the GGJ term in the tearing mode $\Delta^{\prime}$ model (4a), by assuming a finite
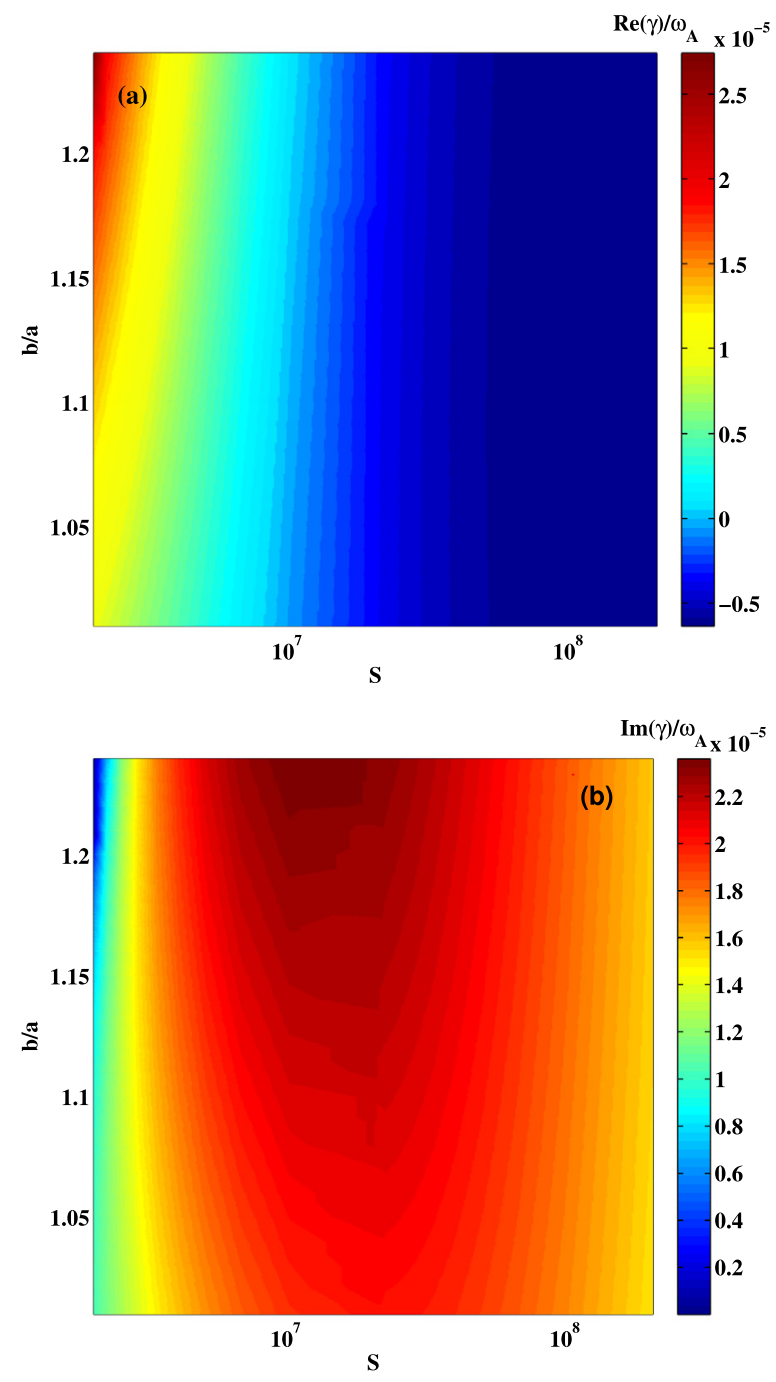

FIG. 6. The (a) growth rate and (b) real frequency of the $\mathrm{n}=1 \mathrm{RWM}$ plotted in $2 \mathrm{D}$ parameter space of the wall position $b / a$ and the Lundquist number $S$. The favorable curvature stabilization effect is included by assuming a finite value for the resistive interchange index $D_{R}=-0.003$. The plasma rotation frequency is assumed to be zero. No drift kinetic damping from trapped EPs is included. negative value for $D_{R}$. The resistive interchange index, which is roughly proportional to the plasma pressure at the rational surface, is normally negative for tokamak equilibria. Figure 5 compares the eigenvalues of the RWM, assuming various values for $D_{R}$. Whilst the mode frequency does not exercise a substantial modification by the GGJ effect, the stability of the mode does qualitatively change. The GGJ term tends to stabilize the RWM, a conclusion also reached in Ref. 30.

The results reported in Fig. 5 are obtained at a relatively small Lundquist number $S=10^{6}$. The stability window in the $b / a$ space is significantly wider with increasing $S$ value and in the presence of finite GGJ term, as shown in Fig. 6, where a 2D parameter scan is performed, varying both the wall distance and the Lundquist number. Note that the mode stabilization, shown in Fig. 6(a), is achieved solely by the GGJ
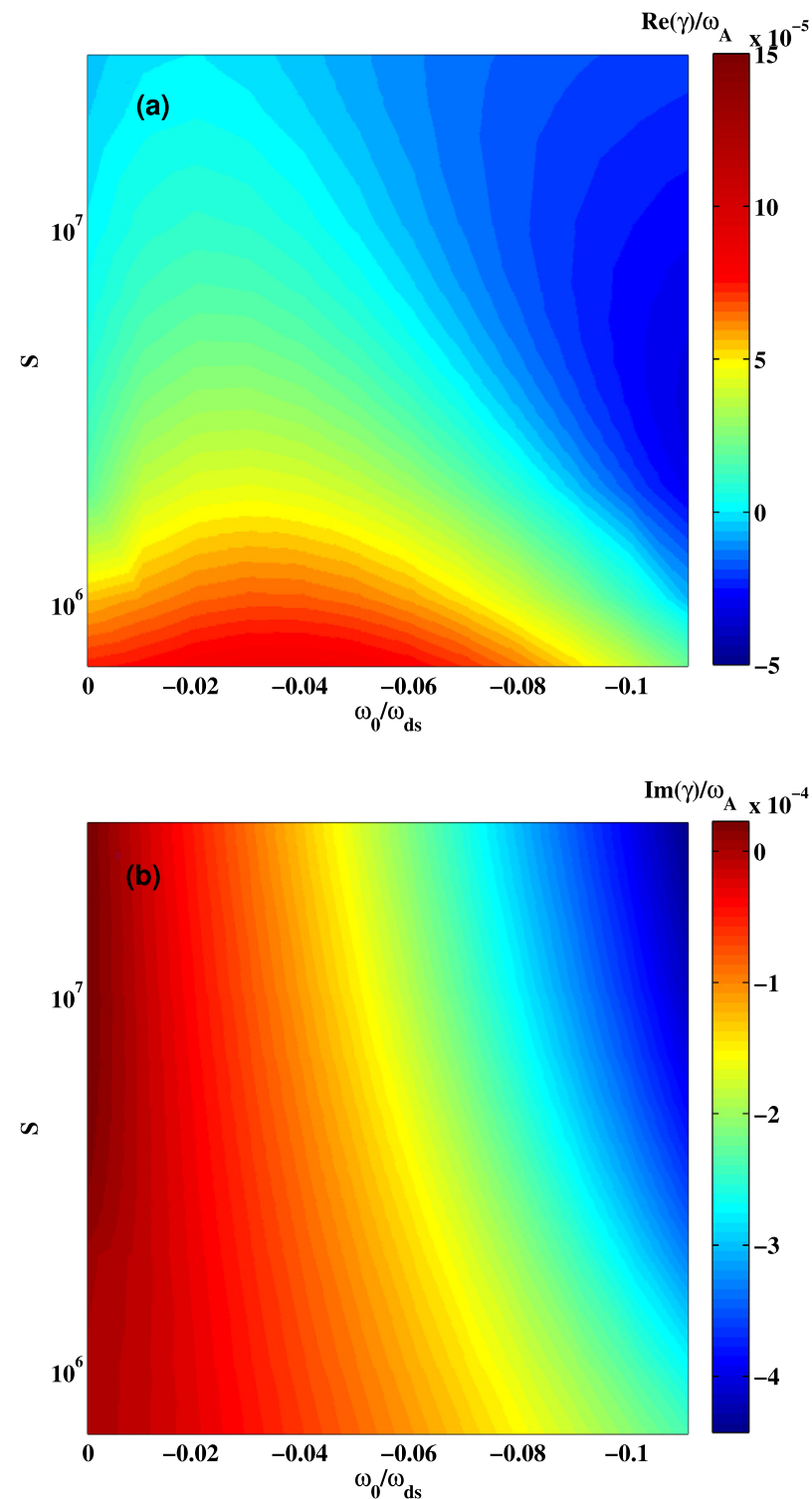

FIG. 7. The (a) growth rate and (b) real frequency of the $\mathrm{n}=1$ RWM plotted in $2 \mathrm{D}$ parameter space of the plasma rotation frequency and the Lundquist number $S$. The resistive wall position is fixed at $b / a=1.2$. The favorable curvature stabilization effect is included by assuming a finite value for the resistive interchange index $D_{R}=-0.003$. No drift kinetic damping from trapped EPs is included. 
stabilization, since the plasma flow velocity is assumed to be zero in this $2 \mathrm{D}$ scan. This also means that the finite mode frequency, shown in Fig. 6(b), is obtained in a static plasma. These two observations (full stabilization and a rotating mode in the absence of the plasma flow) are similar to that occurring to a tearing mode in a toroidal finite beta plasma, when the GGJ effect becomes important. Another interesting observation for Fig. 6 is that at sufficiently large $S$ value, the wall position plays a minor role on the eigenvalue of the RWM.

By fixing the wall position, we make another 2D scan in the parameter space of plasma rotation frequency versus the Lundquist number. The results are shown in Fig. 7. The range of the scanned parameter values as well as all the other plasma parameters are the same as that for Fig. 4, except the additional inclusion of the GGJ in Fig. 7. The key effect is again in the expansion of the stable domain in this $2 \mathrm{D}$ parameter space, introduced by the GGJ stabilization of the RWM.
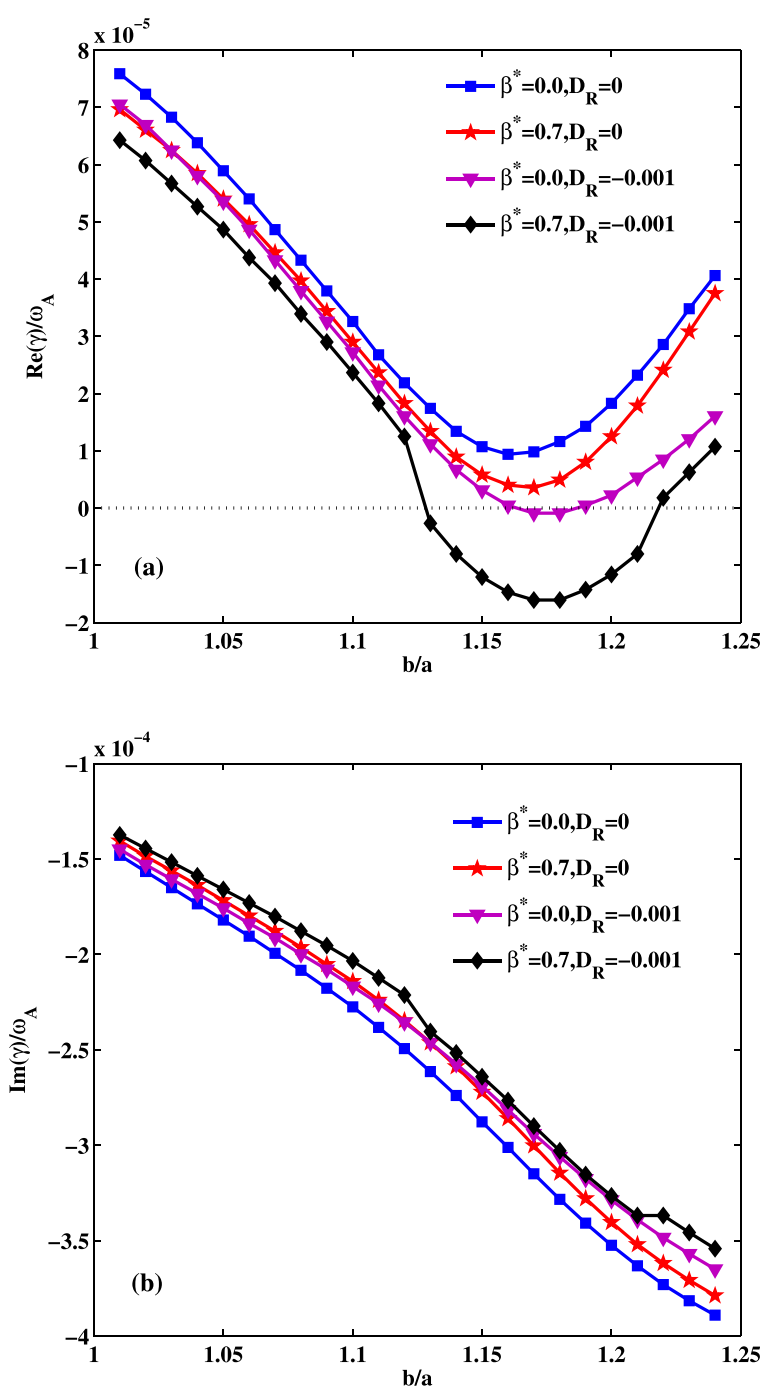

FIG. 8. The (a) growth rate and (b) real frequency of the $\mathrm{n}=1$ RWM versus the minor radius $(b / a)$ of the resistive wall, for four combinations of the energetic particle pressure (normalized by the thermal particle pressure) and the resistive interchange index $D_{R}$. The plasma rotation frequency is fixed at $\omega_{0} / \omega_{d s}=\mathbf{0 . 1}$, and the Lundquist number fixed at $S=2.07 \times 10^{6}$.

\section{Resistive tearing model and drift kinetic model of trapped EPs}

Now we add one more physics into our systematic investigation, namely, the drift kinetic effect from the EPs. In this case, we solve the full dispersion relation (5), where all the perturbed energy terms are finite. We consider only the precessional drift resonance of trapped EPs. The amount of this drift kinetic contribution is measured by the ratio of the energetic ion pressure to the thermal particle pressure, defined as $\beta^{*}$ here. Figure 8 compares the results from four possible combinations: with $\left(\beta^{*}=0.7\right)$ and without $\left(\beta^{*}=0\right)$ EPs kinetic contribution, with $\left(D_{R}=-0.001\right)$, and without $\left(D_{R}=0\right)$ the GGJ effect. Evidently, the GGJ effect and the drift kinetic effect can act in a synergistic manner to reduce the growth rate of the RWM. For the case shown here, the drift kinetic damping, in combination with the GGJ stabilization, helps to open a reasonably large stability window in the $b / a$ space. The real frequency of the mode, however, is not
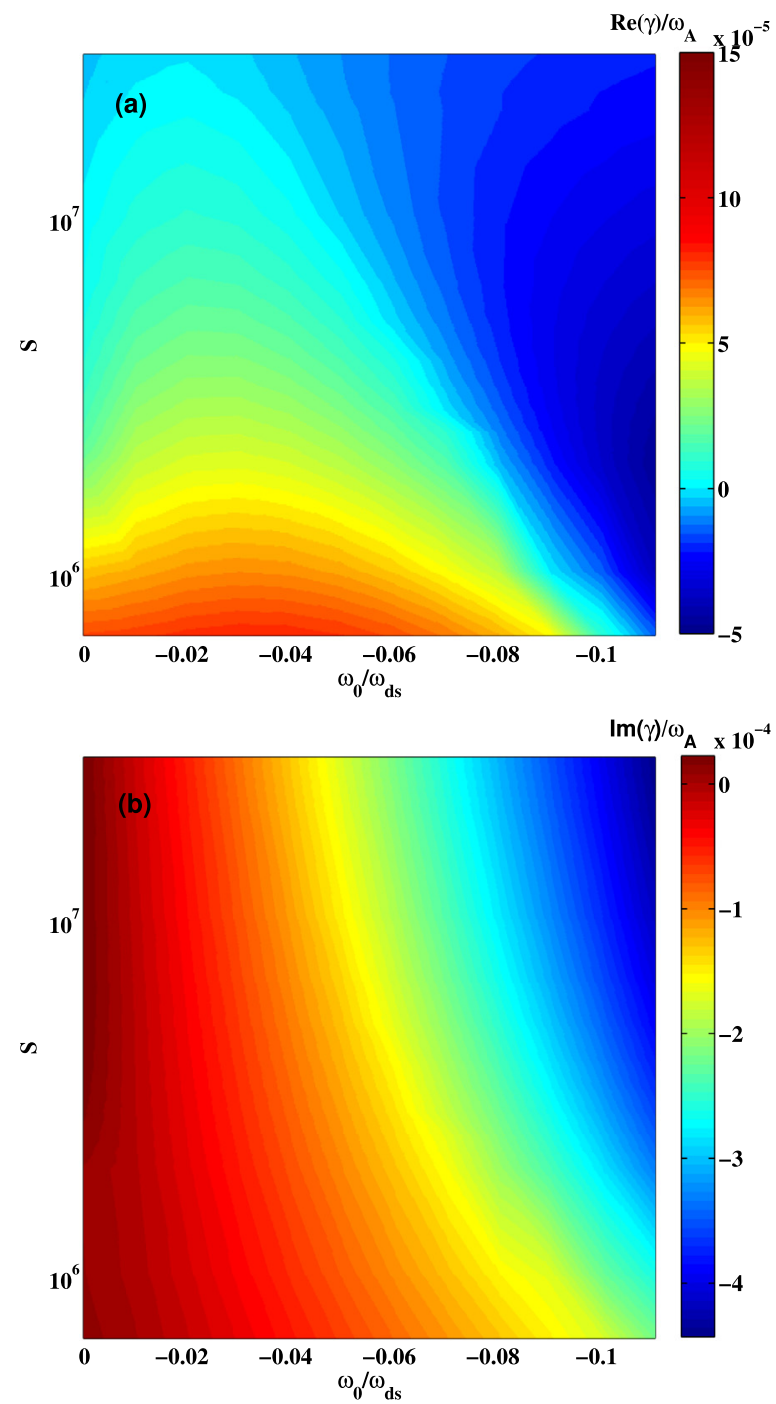

FIG. 9. The (a) growth rate and (b) real frequency of the $\mathrm{n}=1$ RWM plotted in 2D parameter space of the plasma rotation frequency and the Lundquist number $S$. The resistive wall position is fixed at $b / a=1.2$. The favorable curvature stabilization effect is included by assuming a finite value for the resistive interchange index $D_{R}=-0.003$. The drift kinetic damping from trapped EPs is included, with the normalized EPs pressure $\beta^{*}=0.7$. 


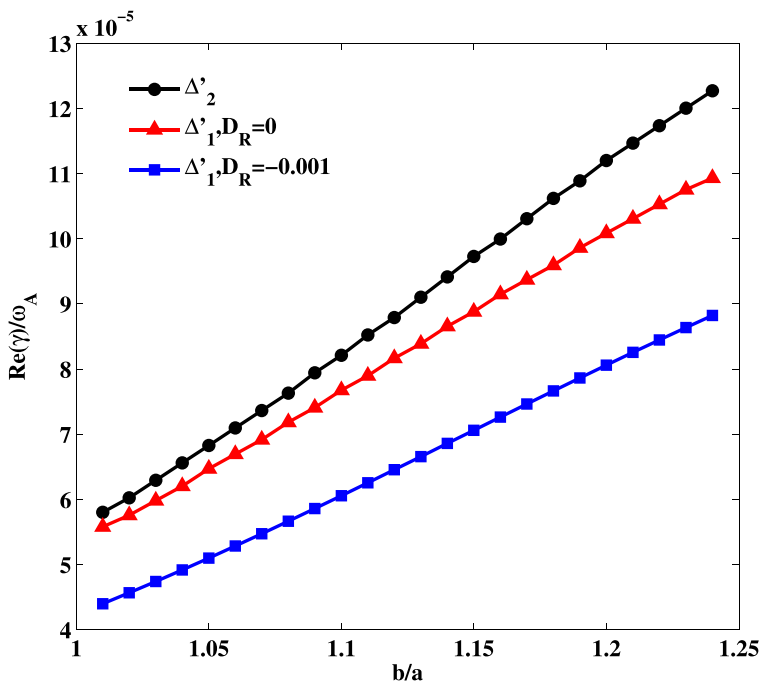

FIG. 10. The growth rate of the $n=1 \mathrm{RWM}$ versus the minor radius $(b / a)$ of the resistive wall, using different models (inner $\Delta^{\prime}$ ) for the resistive layer. The Lundquist number is fixed at $S=2.07 \times 10^{6}$. The plasma rotation frequency assumed to be zero. No drift kinetic effect is included. The frequency of the mode is zero under these conditions.

significantly modified by the aforementioned two effects, as shown in Fig. 8(b).

Figure 8 is obtained by assuming a finite plasma rotation frequency $\omega_{0} / \omega_{d s}=-\mathbf{0 . 1}$. Again, a $2 \mathrm{D}$ scan in the parameter space of both the rotation frequency and the Lundquist number has been performed, but this time in the presence of both the GGJ and the drift kinetic damping. The results are shown in Fig. 9, where all the other plasma parameters are the same in Fig. 7. The stable domain now becomes even wider compared to Fig. 7. Thus, by choosing the same parameter space and by successively adding more physics terms into the model, Figs. 4, 7, and 9 show clear stabilization effects of the (local) favorable average curvature and the (global) drift kinetic damping on the RWM.

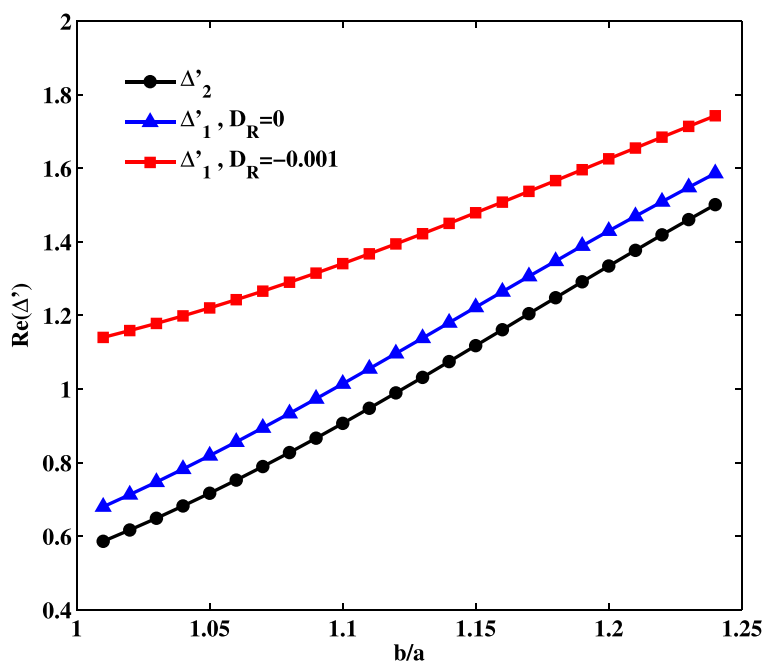

FIG. 11. The real part of the inner $\Delta^{\prime}$, evaluated using the self-consistently determined eigenvalues of the $n=1$ RWM, as shown in Fig. 10, versus the minor radius $(b / a)$ of the resistive wall. The other plasma parameters are the same as in Fig. 10. The imaginary part of $\Delta^{\prime}$ vanishes under these conditions.
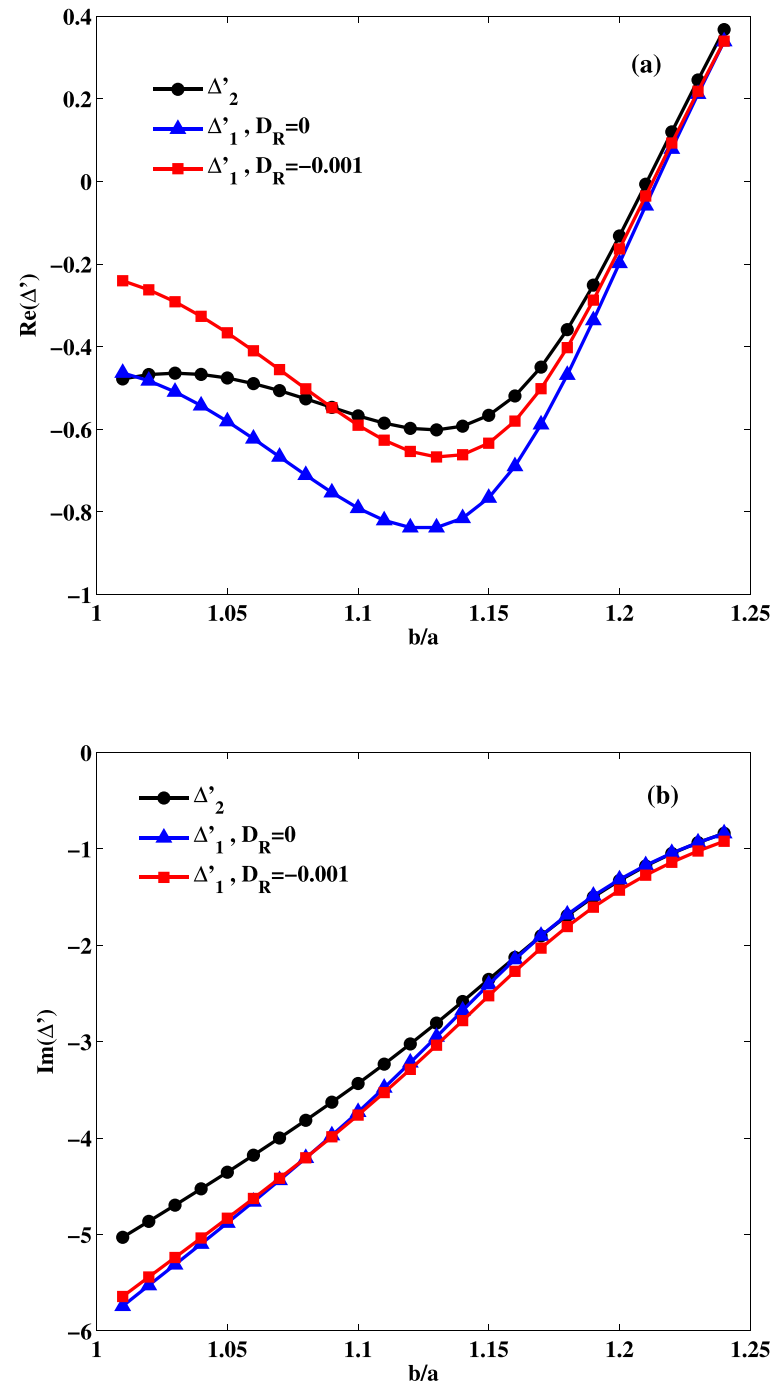

FIG. 12. The (a) real and (b) imaginary parts of the inner $\Delta^{\prime}$, evaluated using the self-consistently determined eigenvalues of the $n=1$ RWM, as shown in Fig. 12, versus the minor radius $(b / a)$ of the resistive wall. The other plasma parameters are the same as in Fig. 12

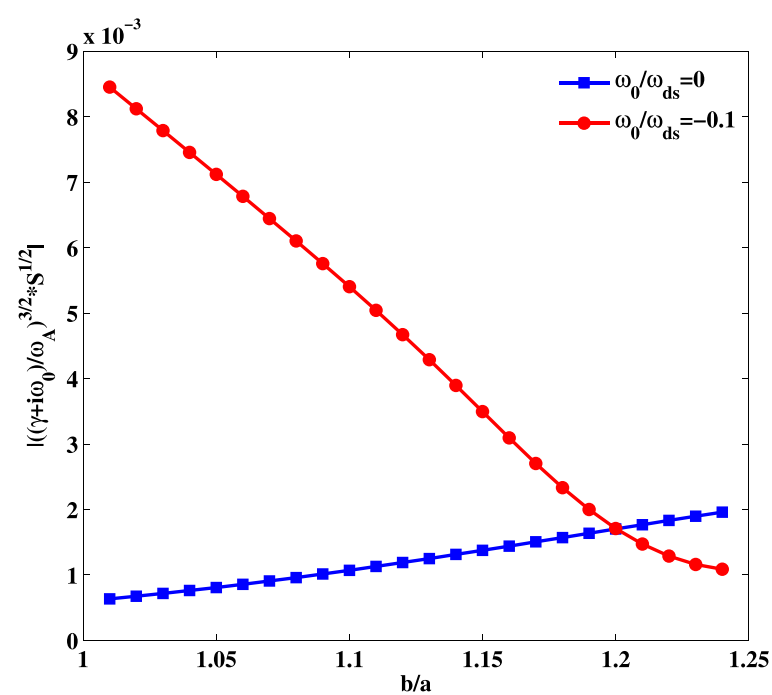

FIG. 13. The absolute value of $\left(\frac{\gamma+i \omega_{0}}{\omega_{A}}\right)^{3 / 2} S^{1 / 2}$ in the $\Delta_{2}^{\prime}$ versus the radial position of the resistive wall for various choices of the plasma rotation frequency. The Lundquist number is fixed at $S=2.07 \times 10^{6}$. No drift kinetic effect is included. 


\section{Comparison of resistive tearing model and resistive kink model}

As a final part of this investigation, in what follows, we compare the predictions on the RWM stability by two different layer models, described by Eqs. (4a) and (4b), respectively. For simplicity, we shall neglect the drift kinetic energy contribution in the dispersion relation (5). We first consider a case with vanishing plasma flow. Figure 10 shows the growth rate of the RWM as function of the wall position, with the resistive tearing mode model $\Delta_{1}^{\prime}\left(D_{R}=\mathbf{0},-\mathbf{0 . 0 0 1}\right)$ and with the resistive kink model $\Delta_{2}^{\prime}$. The real frequency of the mode vanishes for the plasma parameters considered in this case. The resistive kink model results in a more unstable RWM, even when compared with the tearing model without the GGJ stabilization. Associated with this difference are the values of $\Delta_{1}^{\prime}$ and $\Delta_{2}^{\prime}$, shown in Fig. 11 and calculated by inserting the self-constantly obtained RWM eigenvalues from the dispersion (5) back into Eqs. (4a) and (4b), respectively. The real
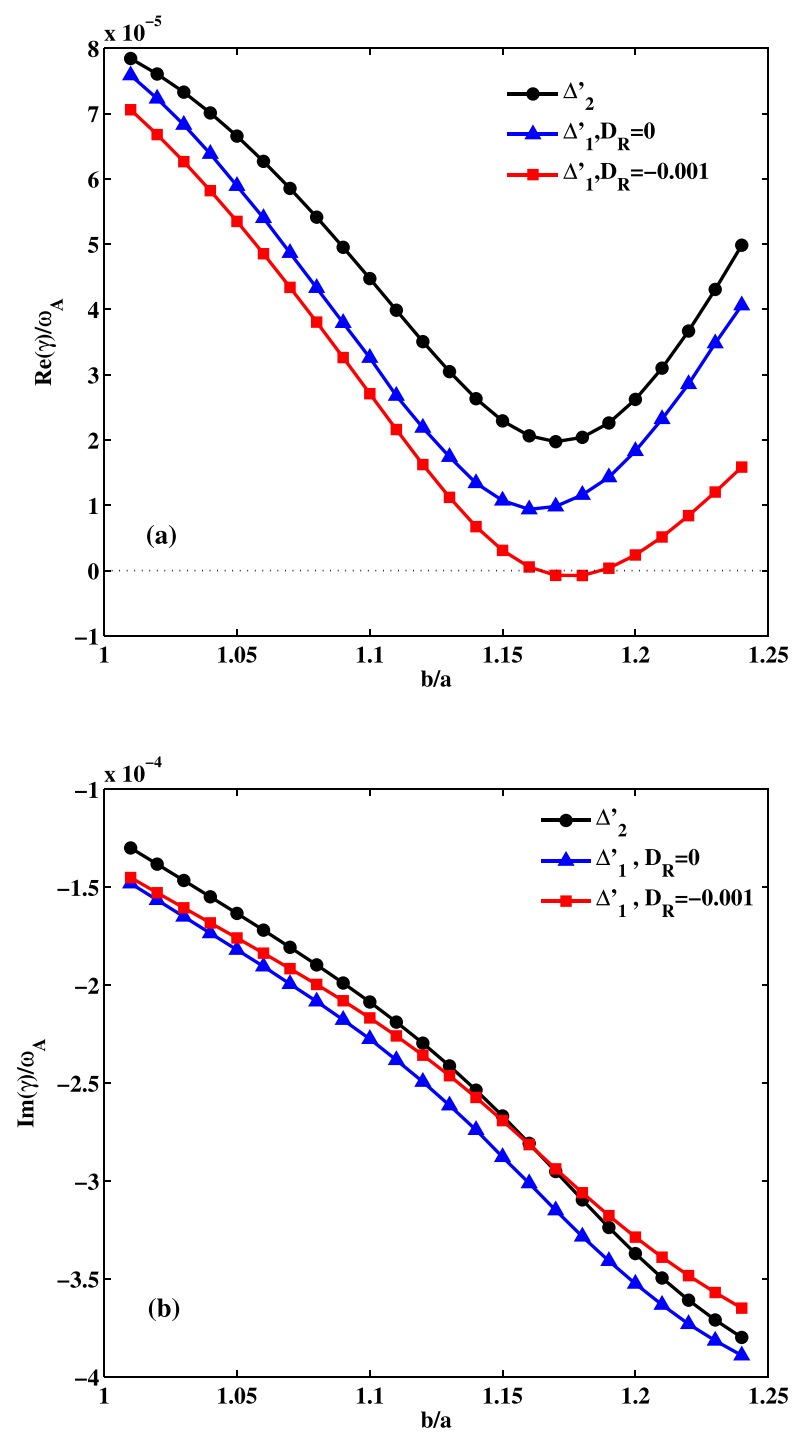

FIG. 14. The (a) growth rate and (b) real frequency of the $n=1 \mathrm{RWM}$ versus the minor radius $(b / a)$ of the resistive wall, using different models (inner $\Delta^{\prime}$ ) for the resistive layer. The Lundquist number is fixed at $S=2.07 \times 10^{6}$. The plasma rotation frequency assumed to be $\omega_{0} / \omega_{d s}=-0.1$. No drift kinetic effect is included. value of $\Delta_{2}^{\prime}$ is smaller than $\Delta_{1}^{\prime}$ (with or without the GGJ effect). Since the strength of the layer energy dissipation is proportional to $\Delta^{\prime}$, less value of $\Delta^{\prime}$ leads to less stability of the RWM, agreeing with the results shown in Fig. 10.

For the next case, we assume a finite plasma flow. The results are shown in Figs. 12 and 13, for the eigenvalue of the mode and the values of $\Delta^{\prime}$, respectively. In this case, both the mode's eigenvalue and the value of $\Delta^{\prime}$ are complex numbers. Again, a general observation is that the resistive kink layer model predicts less stability of the RWM. In order to achieve a better understanding about the reason behind this comparison, we plot the amplitude of the term $\left(\frac{\gamma+i \omega_{0}}{\omega_{A}}\right)^{3 / 2} S^{1 / 2}$ that enters into the Gamma-function of expression (4b), again using the self-consistently obtained eigenvalues of the mode. The results are reported in Fig. 13. Relatively small values of this term correspond to the primarily tearing mode regime. The lack of the GGJ term in the (4b) model is, thus, the primary reason for the larger growth rates of the mode shown in Fig. 14.

\section{CONCLUSION}

We have carried out a systematic investigation of the stability of the RWM, in the presence of both the resistive layer damping, including the toroidal favorable average curvature effect (the GGJ effect), and the drift kinetic damping from trapped energetic ions. The eigenvalue of the mode is obtained by numerically solving the dispersion relation (5), derived for a large aspect ratio circular plasma with simplified equilibrium radial profiles. The stability of the RWM is mapped out in either 1D or 2D parameter spaces involving the plasma resistive (the Lundquist number), the toroidal flow speed, and the distance $b / a$ of the resistive wall to the plasma.

We find that without GGJ effect, a finite plasma resistivity destabilizes the RWM. On the other hand, plasma flow can stabilize the mode in the presence of a finite resistivity, thus opening stability windows in the $b / a$ parameter space, in a similar way as the ideal MHD prediction. The flow stabilization is more effective at large Lundquist number.

The GGJ stabilization, from inside the resistive layer, greatly enhances the RWM stability. In fact, this damping physics alone, without plasma flow and without drift kinetic damping, can result in the full mode stability at large Lundquist number. In the presence of the flow, the stability domain is further widened compared to the cases without the GGJ effect. On the other hand, the GGJ effect generally does not introduce a substantial modification to the real frequency of the mode.

The precessional drift resonance damping of trapped energetic ions acts synergistically with the GGJ effect for the mode stabilization, further widening the stable domain in the 2D parameter space of the plasma flow speed versus the Lundquist number.

We find that the choice of the layer models also affects the prediction for the RWM stability. Generally, the resistive kink layer model, which has previously been assumed in literatures for studying the flow stabilization of the RWM, predicts less stability compared to the resistive tearing mode 
model. This holds even when the GGJ term is excluded from the tearing layer model.

We emphasize that, in this study, the overlap between the drift kinetic effects of EPs and the resistive layer damping physics is excluded. In other words, we assume that the trapped EPs banana orbit width is much greater than the typical resistive layer width. Therefore, the EPs spend little time within the layer, and thus have no significant effect on the inner layer damping. ${ }^{41}$ This situation may be different if thermal particle kinetic effects are included. In such a case, more advanced kinetic models ${ }^{42}$ are needed, in order to better describe the inner layer physics.

\section{ACKNOWLEDGMENTS}

This work was funded by National Natural Science Foundation of China (NSFC) (Grant Nos. 11275041, 11428512, 11405029, and 11105065), and National Magnetic Confinement Fusion Science Program of China with Grant No. 2014GB124004. This work was also part funded by the European Union's Horizon 2020 research and innovation programme under Grant Agreement No. 633053 and from the RCUK Energy Programme [Grant No. EP/ I501045]. The views and opinions expressed herein do not necessarily reflect those of the European Commission.

${ }^{1}$ T. C. Hender, J. C. Wesley, J. Bialek, A. Bondeson, A. H. Boozer, R. J. Buttery, A. Garofalo, T. P. Goodman, R. S. Granetz, Y. Gribov, O. Gruber, M. Gryaznevich, G. Giruzzi, S. Günter, N. Hayashi, P. Helander, C. C. Hegna, D. F. Howell, D. A. Humphreys, G. T. A. Huysmans, A. W. Hyatt, A. Isayama, S. C. Jardin, Y. Kawano, A. Kellman, C. Kessel, H. R. Koslowski, R. J. L. Haye, E. Lazzaro, Y. Q. Liu, V. Lukash, J. Manickam, S. Medvedev, V. Mertens, S. V. Mirnov, Y. Nakamura, G. Navratil, M. Okabayashi, T. Ozeki, R. Paccagnella, G. Pautasso, F. Porcelli, V. D. Pustovitov, V. Riccardo, M. Sato, O. Sauter, M. J. Schaffer, M. Shimada, P. Sonato, E. J. Strait, M. Sugihara, M. Takechi, A. D. Turnbull, E. Westerhof, D. G. Whyte, R. Yoshino, H. Zohm, and the ITPA MHD, Disruption and Magnetic Control Topical Group, Nucl. Fusion 47, S128 (2007).

${ }^{2}$ M. S. Chu and M. Okabayashi, Plasma Phys. Controlled Fusion 52, 123001 (2010).

${ }^{3}$ R. Fitzpatrick and A. Y. Aydemir, Nucl. Fusion 36, 11 (1996)

${ }^{4}$ R. Betti and J. P. Freidberg, Phys. Rev. Lett. 74, 2949 (1995).

${ }^{5}$ C. M. Fransson, B. Lennartson, C. Breitholtz, A. Bondeson, and Y. Q. Liu, Phys. Plasmas 7, 4143 (2000).

${ }^{6}$ A. Bondeson, Y. Q. Liu, C. M. Fransson, B. Lennartson, C. Breitholtz, and T. S. Taylor, Nucl. Fusion 41, 455 (2001).

${ }^{7}$ Y. Q. Liu, A. Bondeson, Y. Gribov, and A. Polevoi, Nucl. Fusion 44, 232 (2004).

${ }^{8}$ J. R. Drake, P. R. Brunsell, D. Yadikin, M. Cecconello, J. A. Malmberg, D. Gregoratto, R. Paccagnella, T. Bolzonella, G. Manduchi, L. Marrelli, S. Ortolani, G. Spizzo, P. Zanca, A. Bondeson, and Y. Q. Liu, Nucl. Fusion 45, 557 (2005).

${ }^{9}$ Y. Q. Liu, I. T. Chapman, M. S. Chu, H. Reimerdes, F. Villone, R. Albanese, G. Ambrosino, A. M. Garofalo, C. G. Gimblett, R. J. Hastie, T. C. Hender, G. L. Jackson, R. J. La Haye, M. Okabayashi, A. Pironti, A. Portone, G. Rubinacci, and E. J. Strait, Phys. Plasmas 16, 056113 (2009).
${ }^{10}$ V. D. Pustovitov, Nucl. Fusion 55, 033008 (2015).

${ }^{11}$ A. Bondeson and D. Ward, Phys. Rev. Lett. 72, 2709 (1994).

${ }^{12}$ M. S. Chu, J. M. Greene, T. H. Jensen, R. L. Miller, A. Bondeson, R. W. Johnson, and M. E. Mauel, Phys. Plasmas 2, 2236 (1995).

${ }^{13}$ R. Betti, Phys. Plasmas 5, 3615 (1998).

${ }^{14}$ B. Hu and R. Betti, Phys. Rev. Lett. 93, 105002 (2004).

${ }^{15}$ Y. Q. Liu, M. S. Chu, I. T. Chapman, and T. C. Hender, Phys. Plasmas 15, 112503 (2008).

${ }^{16}$ I. T. Chapman, C. G. Gimblett, M. P. Gryaznevich, T. C. Hender, D. F. Howell, Y. Q. Liu, and S. D. Pinches, Plasma Phys. Controlled Fusion 51, 055015 (2009).

${ }^{17}$ Y. Q. Liu, M. S. Chu, I. T. Chapman, and T. C. Hender, Nucl. Fusion 49, 035004 (2009).

${ }^{18}$ J. W. Berkery, S. A. Sabbagh, H. Reimerdes, R. Betti, B. Hu, R. E. Bell, S. P. Gerhardt, J. Manickam, and M. Podestà, Phys. Plasmas 17, 082504 (2010).

${ }^{19}$ Y. Q. Liu, I. T. Chapman, J. P. Graves, G. Z. Hao, Z. R. Wang, J. E. Menard, M. Okabayashi, E. J. Strait, and A. Turnbull, Phys. Plasmas 21 , 056105 (2014).

${ }^{20}$ G. Z. Hao, Y. Q. Liu, A. K. Wang, H. B. Jiang, G. Lu, H. D. He, and X. M. Qiu, Phys. Plasmas 18, 032513 (2011).

${ }^{21}$ C. Liu, Y. Q. Liu, Y. Liu, G. Z. Hao, L. Li, and Z. R. Wang, Nucl. Fusion 55, 063022 (2015).

${ }^{22}$ Z. R. Wang, M. J. Lanctot, Y. Q. Liu, J. K. Park, and J. E. Menard, Phys. Rev. Lett. 114, 145005 (2015).

${ }^{23}$ Y. Q. Liu, A. Bondeson, M. S. Chu, J. Y. Favez, Y. Gribov, M. Gryaznevich, T. C. Hender, D. F. Howell, R. J. L. Haye, J. B. Lister, P. d. Vries, and E. J. Contributors, Nucl. Fusion 45, 1131 (2005).

${ }^{24}$ M. Takechi, G. Matsunaga, N. Aiba, T. Fujita, T. Ozeki, Y. Koide, Y. Sakamoto, G. Kurita, A. Isayama, and Y. Kamada, Phys. Rev. Lett. 98, 055002 (2007)

${ }^{25}$ M. J. Lanctot, H. Reimerdes, A. M. Garofalo, M. S. Chu, Y. Q. Liu, E. J. Strait, G. L. Jackson, R. J. La Haye, M. Okabayashi, T. H. Osborne, and M. J. Schaffer, Phys. Plasmas 17, 030701 (2010).

${ }^{26}$ G. Z. Hao, Y. Q. Liu, A. K. Wang, and X. M. Qiu, Phys. Plasmas 19, 032507 (2012).

${ }^{27}$ J. M. Finn, Phys. Plasmas 2, 198 (1995).

${ }^{28}$ J. M. Finn, Phys. Plasmas 2, 3782 (1995).

${ }^{29}$ A. Bondeson, C. G. Gimblett, and R. J. Hastie, Phys. Plasmas 6, 637 (1999).

${ }^{30}$ Y. L. He, Y. Q. Liu, Y. Liu, G. Z. Hao, and A. K. Wang, Phys. Rev. Lett. 113, 175001 (2014).

${ }^{31}$ A. Bondeson and H. X. Xie, Phys. Plasmas 4, 2081 (1997)

${ }^{32}$ S. W. Haney and J. P. Freidberg, Phys. Fluids B 1, 1637 (1989).

${ }^{33}$ Y. Q. Liu, M. S. Chu, C. G. Gimblett, and R. J. Hastie, Phys. Plasmas 15, 092505 (2008).

${ }^{34}$ R. B. White, The Theory of Toroidally Confined Plasmas, Second ed. (Imperial College Press, 2001), p. 159.

${ }^{35}$ A. H. Glasser, J. M. Greene, and J. L. Johnson, Phys. Fluids 18, 875 (1975).

${ }^{36}$ Y. Q. Liu, J. W. Connor, S. C. Cowley, C. J. Ham, R. J. Hastie, and T. C. Hender, Phys. Plasmas 19, 072509 (2012).

${ }^{37}$ B. Coppi, R. Galvao, R. Pellat, M. N. Rosenbluth, and P. H. Rutherford, Fiz. Plazmy 2, 961 (1976) [Sov. J. Plasma Phys. 2, 533 (1976)].

${ }^{38}$ T. M. Antonsen, Jr. and Y. C. Lee, Phys. Fluids 25, 132 (1982).

${ }^{39}$ H. Biglari and L. Chen, Phys. Fluids 29, 1760 (1986).

${ }^{40}$ G. Z. Hao, A. K. Wang, Y. Q. Liu, and X. M. Qiu, Phys. Rev. Lett. 107, 015001 (2011).

${ }^{41}$ F. Porcelli, R. Stankiewicz, W. Kerner, and H. L. Berk, Phys. Plasmas 1, 470 (1994)

${ }^{42}$ J. W. Connor, R. J. Hastie, and A. Zocco, Plasma Phys. Controlled Fusion 54, 035003 (2012). 